\section{Plenary sessions}

\section{P1 CLOSING THE AUDIT LOOP. OUTCOME OF SECOND NATIONAL AUDIT OF THE MANAGEMENT OF ACUTE ITP-A CHANGE IN PRACTICE}

P.H.B. Bolton-Maggs, I. Moon. Department of Haematology, Alder Hey Children's Hospital, Liverpool L12 2AP

The management of childhood immune thrombocytopenic purpura (ITP) is controversial. Children present with dramatic skin signs but even with a very low platelet count serious bleeding is rare. The disease remits spontaneously in most within 8 weeks. The first audit of national practice against published guidelines demonstrated significant variation and non-compliance. An important feature of the survey (1995-6) was the clinical classification of children by their physicians by symptoms as 'mild', 'moderate', or 'severe', ignoring the platelet count. 425 cases (over 14 months) were analysed of whom only 13 $(3 \%)$ had severe bleeding, usually epistaxis or Gl bleeds; $76 \%$ had clinically mild ITP even with the majority of counts less than $20 \times 10^{\circ} /$. There were no deaths or intracranial haemorrhages (ICH). 60\% of children were given treatment to raise the count, with IVIG used excessively $(23 \%)$ against the guideline standard. $40 \%$ were managed without specific therapy. In 2000 the audit was repeated over 12 months. 305 questionnaires were analysed giving a population with very similar characteristics to the first survey, but slightly lower case rate (about 25-30 per month). Only 5\% were classified as severe by symptoms although $89 \%$ had presenting counts of $<10 \times 10^{\circ} /$. Two children had $\mathrm{ICH}$ with almost complete recovery and there were no deaths. A significant change in practice was observed with a move towards 'no treatment' in $63 \%$ (from $40 \%$ ) and a reduction in the proportion having marrow examination from $168 / 425(40 \%)$ to $55 / 305$ $(18 \%)$ mainly because of the reduction in treatment. If all children with clinically mild ITP were not treated, $74 \%$ would avoid therapy. These national surveys confirm that ITP in children is a benign disease with an incidence of $\mathrm{ICH}$ of 2 in $730(0.3 \%)$, that more children can be managed expectantly without specific therapy but with good advice, and that clinicians can be persuaded to change their practice as a result of audit when there is appropriate evidence and education.

\section{P2 TEN-YEAR TRENDS IN RISK FACTORS FOR CORONARY HEART DISEASE IN NORTHERN IRISH ADOLESCENTS: THE N.I. YOUNG HEARTS PROJECT 2000}

D.C. Watkins, L.J. Murray, I.S. Young, C.A.G. Boreham, G. Cran, P.J. Robson, J.M. Savage. Dept. of Child Health, Queens University of Belfast, Institute of Clinical Science, Belfast BT12 6JB

Introduction: In 1989-90, the first NI Young Hearts Project (YH1) reported the incidence of major cardiovascular risk factors in 1015 12 and 15 year-olds. Results justified concern regarding future disease in this population, within a province with consistently high CHD prevalence. A 10-year cross-sectional follow-up study (YH2000) set out to evaluate secular trends in these risk factors in an equivalent cohort of Northern Irish schoolchildren.

Methods: A randomly selected demographically representative $3.6 \%$ province population sample of 2017 children aged 12 and 15 years was studied to obtain information on body composition, blood pressure, lipid profile, cigarette smoking, physical activity, cardiorespiratory fitness, dietary intake and family history, replicating the methodology of $\mathrm{YH} 1$.

Results: Univariate analysis demonstrated the following comparisons of means: height increased significantly in all 4 age groups $(\mathrm{p}<0.0001)$, as did weight in all groups except 15 year-old boys. Body mass index showed a significant increase only in 12 year-old girls $(p=0.0002)$. Percentage of body fat, calculated using skinfold thickness measurements, also increased significantly in girls but not boys. Both systolic and diastolic blood pressure showed highly significant decreases $(p<0.0001)$ across all groups. Total cholesterol decreased significantly in 15 year-old boys $(p=0.03)$ and cholesterol:HDL ratio increased in 12 year-old girls $(p=0.03)$; no other lipid profile changes reached significance. Maximal aerobic fitness increased significantly in boys ( $p<0.01)$ but not girls. Total energy intake increased in 12 year-old girls $(p=0.01)$, while total intake of fat and fat subgroups showed a decreasing tendency in all groups

Conclusions: Children of both sexes are bigger for age than ten years ago, and girls tend to be fatter. Among other major CHD risk factors, blood pressure and dietary fat intake show improvements in both sexes, and aerobic fitness has improved in boys. It may be, therefore, that public health messages regarding $\mathrm{CHD}$ prevention are having an impact in Northern Irish secondary school-age children.

\section{P3 A RANDOMISED CONTROLLED TRIAL OF AN EVIDENCED BASED WEANING STRATEGY DESIGNED SPECIFICALLY TO MEET THE NEEDS OF PRETERM} INFANTS

K.D. Foote, L.D. Marriott', J.A. Bishop', A.C. Kimber', J.B. Morgan'. Royal Hampshire County Hospital, Winchester SO22 5DG; 'University of Surrey, Guildford GU2 5XH

Post discharge nutrition in general and weaning in particular are important but neglected areas for research. Preterm infants have additional nutrient needs to replenish tissue stores and support catch-up growth. No account of these additional needs is made in current recommendations for weaning. We designed a weaning strategy based on current evidence, calculated needs and expert opinion. To evaluate the strategy we undertook a study of 68 preterm infants, mean birth weight 1.47 (SD 0.43) kg and mean gestational age 31.3 (SD 2.9) weeks randomised to either an early weaning with nutrient enhanced solids group (A) or a current best practice group (B). Blood samples were collected at 0 and 6 months, anthropometry was undertaken (by one observer, LDM) at $0,6,12$ and 18 months and psychometric testing carried out at 18 months gestation corrected age. At 6 months GCA mean haemoglobin and serum iron levels were significantly higher in group $A(12 \mathrm{~g} / \mathrm{dl}, 12.2 \mathrm{umol} / \mathrm{l})$ than in group $B(11.4 \mathrm{~g} / \mathrm{dl}$, 9.4 umol/l) $p=0.01$ and 0.006 respectively. In addition, the mean haemoglobin for the intervention group increased significantly during the study, whilst the mean serum iron of the control group decreased significantly, $p=0.001$ and 0.004 respectively. Over the first 12 months, significantly higher mean length growth velocity was seen in group $A$ than in group $B, 5.1$ versus $4.9 \mathrm{~mm} /$ week, $p=0.04$. At 18 months GCA, group A had on average a 5 point advantage in mental scores and 7 point advantage in behaviour rating compared to group B on Bayley scales, $p=n s$. Preterm infants born in developed countries should be weaned from 3 months of postnatal age with solid foods of a higher nutrient density than currently recommended.

\section{P4 POST ASPHYXIAL HYPERTHERMIA-DAMAGES THE NEONATAL BRAIN}

Thoresen $\mathrm{M}^{1}$, Tooley $\mathrm{J}^{1}$, Satas $\mathrm{S}^{1}$, Eagle $\mathrm{R}^{2}$, Cherian $\mathrm{S}^{1}$, Porter ${ }^{3}$. 'Dept of Child Health, St Michaels Hospital, University of Bristol; ' 2 Dept of Anatomy, School of Veterinary Science, University of Bristol; ${ }^{3}$ Dept of Pathology, St Michaels Hospital, University of Bristol, UK

Background: Apart from general supportive measures, few therapeutic options are available outside multicentre trials for the treatment of neonatal Hypoxic Ischaemic (HI) injury. Maintenance of normothermia has not been stressed as an important factor in the management of these babies, despite growing evidence that hyperthermia (HT) may be damaging.

Aim: To determine whether a short period of $\mathrm{HT}$ is damaging to the neonatal rat brain, either immediately or 24 hours after an $\mathrm{HI}$ insult.

Method: 97 Wistar rats (7 days old (P7)) underwent Left Carotid artery ligation and were randomised into one of four groups; earlyHT, lateHT, Normothermia (NT) or shamHT. All groups except the shamHT group were then put into a chamber of $8 \%$ oxygen for $2 \mathrm{hrs}$. Immediately after this the earlyHT group along with the shamHT group were transferred into a hyperthermic chamber to maintain rectal temperature $(\mathrm{Tr})$ at $40^{\circ} \mathrm{C}$ for 4 hours. The NT group and lateHT group maintained normothermia $\left(\operatorname{Tr} 37.5^{\circ} \mathrm{C}\right)$ for 4 hours in another chamber. The rats were then returned to the dam. $24 \mathrm{~h}$ after the insult the lateHT group were transferred into a chamber and kept at $\operatorname{Tr} 40^{\circ} \mathrm{C}$ for $4 \mathrm{~h}$ with the remaining rats kept at $\operatorname{Tr} 37.5^{\circ} \mathrm{C}$. One animal in each chamber carried a rectal temperature probe however they were not used in 
further analysis. At P14 the brains were perfusion fixed under terminal anaesthesia and sections from 4 areas per brain prepared for histological analysis by a perinatal pathologist (HP) using our 0 (no damage) - 4 (>75\% damage) score. Animals dying between P8 and P14 were also perfusion fixed and examined.

Results: Neuropathology damage scores were highest in the earlyHT group 15.5 (0.9) (mean +/- (SE)) vs the lateHT group 12.5 (1.4), NT group $11.6(1.3)$ and the shamHT group O(0). The earlyHT score was significantly higher than both the NT $(p=0.007)$ and ShamHT ( $p=0.0001)$. There was no significant difference between the neuropathology scores in the lateHT and NT groups.

Conclusions: Early postinsult hyperthermia increases neurological damage in the neonatal rat brain. Great care should be taken to avoid inadvertent or deliberate hyperthermia after $\mathrm{HI}$ injury in the newborn.

\section{P5 INCIDENCE OF SEVERE RETINOPATHY OF PREMATURITY AND TRENDS IN SURVIVAL OF INFANTS WITH BIRTH WEIGHT 1250 GRAMS OR LESS}

B.A. Hameed, K. Shyamanur, D. Field, B. Manktelow, GWoodruff, S. Kotecha, E.S. Draper. Neonatal Intensive Care Unit, Leicester Royal Infirmary, Leicester, UK

Aims: To examine the trends in survival rate and the incidence of severe( $\geqslant$ Grade 3) Retinopathy of Prematurity(ROP) in infants with birth weight of $\leqslant 1250$ grams in a geographically defined population over a ten year period.

Methods: A retrospective study of all infants born to mothers resident in Leicestershire county, United Kingdom, with birth weight $\leqslant$ 1250 grams, during the period January 1990 to December 1999. Cases were identified by the Trent Neonatal Survey. The survival rate and incidence of severe ROP were compared between infants born in the two successive five year periods from 1990 to 1994 and 1995 to 1999

Results: In the second five year block (1995-1999), when compared to the first (1990-1994), whilst the total number of live births fell (56564 v 60789), there was a significant increase in the number of births (both live and dead; $734 \vee 615 ; p<0.0001$ ) and live births $(556$ v $455 ; p<0.0001)$ with birth weight $\leqslant 1250$ grams. An identical proportion of the live births $(71 \%)$ was admitted for intensive care, but survival to 42 weeks adjusted gestation was significantly better in the latter time period lodds ratio $0.54[95 \% \mathrm{Cl} 0.39$ to 0.75 ] $p=0.0002$ ). The number of cases of severe ROP were high during the second time period compared to the first $(36 \vee 9)$. A significantly increased risk for the development of severe ROP was seen during the second time period lodds ratio $2.92[95 \% \mathrm{Cl} 1.37$ to 6.20$]$ $\mathrm{p}=0.0054$ ). Improved survival had however resulted in an increased number of low birth weight infants reaching the stage at which they were eligible for eye examination. The odds ratio was hence corrected for the risk of ROP to take account of this change in birth weight and gestation but the increased risk remained during the second five year period(odds ratio 2.81 [95\% Cl 1.27 to 6.21$] \mathrm{p}=$ $0.011)$.

Conclusions: There is strong evidence that the survival rate and incidence of severe ROP among babies with birth weight $\leqslant 1250$ grams, have increased in the latter half of the last decade. The increased risk of severe ROP among survivors remains even after adjusting for the weight and gestation at birth.

\section{P6 INCIDENCE OF PERICARDIAL EFFUSION/CARDIAC TAMPONADE ASSOCIATED WITH NEONATAL PERCUTANEOUS LONG LINES}

K. Beardsall, D.K. White, A.W.R. Kelsall. Neonatal Intensive Care Unit, Rosie Hospital, Cambridge UK

Introduction: Percutaneous long lines (PLL) are widely used in neonatal intensive care to provide secure vascular access for the administration of parentral nutrition. Different complications have been reported with pericardial effusion/tamponade the most serious and potentially fatal. We aimed to determine the frequency of pericardial effusion/tamponade associated with neonatal PLLs.

Methods: A 5 year retrospective postal survey. Questionnaires were sent to the lead neonatal paediatrician in all UK hospitals providing neonatal care.

Results: 243 questionnaires were sent and 189 returned (79\%). $165(87 \%)$ of the responding units inserted PLLs. We estimate that 10000 lines are inserted annually in the UK. All units checked the position of PLLs by $x$ ray, only $45(27 \%)$ routinely used contrast. One hundred and twenty six (76\%) aimed to position the PLL tip in the vena cavae, outside the heart, and $25(15 \%)$ aimed for the right atrium. Of the 79 cases of pericardial effusion/cardiac tamponade identified, complete information was available in 77 cases. Of these $48162 \%$ infants survived. Of the 29 infants who died, diagnosis was only made at postmortem in 20. In 57 infants a clinical diagnosis was made, it was confirmed by echocardiography in $37(65 \%)$, and pericardial tap was attempted in $44(77 \%)$. Only 9 of these babies died $(16 \%)$. We estimate that 1.7 pericardial effusion/cardiac tamponades will occur for every 1,000 lines inserted, with a fatality rate of 0.6 per 1,000 PLLs inserted.

Conclusions: The incidence of pericardial effusion/cardiac tamponade is low. If this condition is considered when an infant with a PLL deteriorates and appropriate treatment initiated mortality can be substantially reduced.

\section{P7 THE COMPLEXITY OF THE INFLAMMATORY RESPONSE TO MENINGOCOCCAL SEPSIS REVEALED BY GENE EXPRESSION PROFILING USING CDNA MICROARRAYS}

N. Pathan', C. Hemingway', A. Goodsall', S. Welch', D. Relman², M. Levin'. 'Department of Paediatrics, Imperial College School of Medicine, ${ }^{2}$ Department of Infectious Diseases, Stanford University School of Medicine

Aims: Attempts to understand the pathogenesis of sepsis have focused on a limited number of mediators, such as TNF $\alpha$ and IL-1 $\beta$. The initial immune response resulting from sepsis cascades into the production of numerous chemokines and cytokines and other signalling molecules, many of which have not been characterised. The development of gene array technology provides the possibility of simultaneously studying not just single inflammatory mediators, but the entire repertoire. We postulated that these methods would provide new insights into the mechanisms involved in sepsis.

Methods: RNA was isolated from blood samples taken on admission of patients with meningococcal sepsis. The messenger RNA was amplified. Fluoresecent cDNA probes were prepared from these samples, and from a reference mRNA prepared from normal lymphoid cell lines, by reverse transcription'. In addition, whole blood obtained from healthy volunteers was inoculated with live meningococci, and RNA recovered at sequential timepoints. The fluorescent probes were hybridized to a DNA microarray containing 30000 genes expressed in lymphoid and macrophage cell lines. The relative fluorescent intensity of the sample mRNA compared to the reference mRNA was measured using a fluorescent scanner'

Results: Whole blood stimulation in vitro generated a gene expression pattern that was largely anticipated, with high expression levels of TNF $\alpha$, IL-6, IL- $1 \alpha$, and IL-1 $\beta$. In addition smaller chemokines and cytokines were also highly expressed, such as MIP, and the small inducible cytokine subfamily. In contrast, patient gene expression demonstrated a markedly different profile, with a significant upregulation of intracellular signalling molecules, such as mitogen activated protein kinase, nuclear factor kappa B, matrix metalloproteinase and guanine nucleotide binding protein

Conclusions: Using microarray technology, this study is the first to investigate gene expression profiles in the blood of patients with meningococcal sepsis and to compare it with in vitro stimulation on an unprecedented scale. The findings will provide important insights into the pathogenesis of septic shock, and the highly complex nature of the host response.

1. Alizadeh, et al. Nature. 2000;403:503-1 1 .

\section{P8 MATERNAL NUTRITIONAL MANIPULATION OF INSULIN-LIKE GROWTH FACTOR -I RECEPTOR MRNA ABUNDANCE AND FETAL ADIPOSE TISSUE DEPOSITION}

T. Stephenson, J. Bispham, J. Dandrea, P.C. Tong', S. Gilmour', P.D. Gluckman', M.E. Symonds. Academic Division of Child Health, School of Human Development, University Hospital, Nottingham NG7 2UH; 'Liggins Institute for Medical Research, University of Auckland, New Zealand 92019

Aims: Insulin-like growth factor (IGF)-I, acting through class I cytokine receptors $(R)$, regulates fetal growth and may also control adipose tissue deposition. Postnatal treatment of rat pups, born to nutrient restricted mothers, with IGF-I alleviates obesity. It is not known whether maternal nutrition programmes adipose tissue deposition or IGF-IR abundance.

Methods: Twelve singleton-bearing ewes of similar body weight and parity were entered into the study. Six ewes were provided with $60 \%$ of their total metabolisable energy (ME) requirements for body 
weight and pregnancy from 28 to $80 \mathrm{~d}$ gestation (i.e. nutrient restricted (NR)) whilst the remainder consumed $150 \%$ of ME requirements (i.e. well-fed (WF)). After $80 \mathrm{~d}$ gestation, all ewes received $100 \%$ of ME requirements. Between 140-145 days gestation (term $=148$ days) fetal perirenal adipose tissue was sampled and total RNA extracted. Oligonucleotide primers for the IGF-IR were designed for use in RT-PCR and generated a 498bp PCR product. Results are expressed in arbitrary units (a. u.) as a ratio of an 18S rRNA internal control as means with their standard errors.

Results: Maternal nutrient restriction during early-to-mid gestation increased the abundance of IGF-IR mRNA (WF: $5.3 \pm 0.9$; NR: $9.0 \pm 1.2$ a.u. $(\mathrm{P}<0.05))$ in conjunction with more adipose tissue deposition (WF: 21.6 \pm 0.9 ; NR: $26.6 \pm 0.7 \mathrm{~g}(\mathrm{P}<0.05))$ at term.

Conclusion: Maternal nutrient restriction during early to mid gestation promotes adipose tissue deposition in conjunction with an increase in IGF-IR mRNA abundance. These findings suggest that fetal adipose tissue development is programmed by maternal nutrition during pregnancy and may contribute to obesity in later life.

\section{P9 DO HARM REDUCTION STRATEGIES REDUCE INFANT SMOKE EXPOSURE?}

R.J.D. Moy, C. Blackburn, N.J. Spencer, A. Dolan, S. Bolas, C. Coe. Coventry and Birmingham

Objective: to study the relationship between parent-reported harm reduction strategies and infant smoke exposure measured by urinary cotinine:creatinine ratio (uccr).

Study design: cross-sectional survey.

Setting: Coventry and Birmingham.

Main outcomes: log transformed infant uccr.

Participants: 314 infants in smoking households were enrolled in the study by the family health visitor at the primary visit. The data presented here relate to 123 infants for whom data were available on infant uccr, main carer's salivary cotinine, partner's average daily cigarette consumption, housing tenure, overcrowding, and current feeding method.

Results: the mean log infant uccr was lower for infants whose parents reported strict measures $(1.44(95 \% \mathrm{Cl} 0.94,1.94))$ compared with none/less strict $(2.60(95 \% \mathrm{Cl} 2.40,2.80))$. In linear regression models fitted on log uccr, reported use of strict harm reduction measures was independently associated with a small reduction in infant uccr $(B=-0.63(95 \% \mathrm{Cl}-1.26,-0.25))$ after adjusting for main carer's salivary cotinine level, partner's average daily cigarette consumption, housing tenure, overcrowding and infant feeding method. The final model accounted for $50.7 \%$ of the variance in infant uccr. When breast feeding infants were excluded, harm reduction showed a reduction in infant uccr $(B=-0.99(95 \% \mathrm{Cl}-1.59,-0.39))$ and final model accounted for $33.0 \%$ of the variance.

Conclusions: parent-reported strict harm reduction measures (banning smoking within the house) were associated with a small but significant reduction in an objective measure of infant smoke exposure independent of potential confounding variables. Our results suggest that less strict harm reduction measures such as not smoking in the same room as the infant have no effect on infant cotinine levels.

\section{P10 HOW LONG SHOULD WE OBSERVE CHILDREN AFTER HEAD INJURY IN HOSPITAL: ARE CURRENT GUIDELINES APPROPRIATE?}

A.L. Curran, P.M. Sharples. Paediatric Neurology Research Group, Frenchay Hospital, Bristol BSI6 ILE

Introduction: Traumatic brain injury (TBI) is a major cause of admission to hospital in children of $>1$ year. Admission for observation for 24 hours was previously considered necessary because of the risk of developing delayed, treatable complications, namely intracranial haemorrhage $(\mathrm{ICH})$ and diffuse brain swelling, which usually manifest as a secondary deterioration in level of consciousness ( $2^{\circ}$ LOC). However, on the basis of data concerning the risk of intracranial haemorrhage following paediatric $\mathrm{TBI}$ in Wales, Sainsbury and Sibert suggested in 1984 that children can be safely discharged 6 hours after injury provided that they have no abnormal neurological signs or symptoms.

Aims: i) To identify children who developed a secondary deterioration in LOC following TBI requiring admission to ICU and ascertain the cause; ii) To determine whether or not these patients had abnormal signs or symptoms at 6 hours after injury.

Methods: Retrospective case notes review of all TBI children to the Regional Neurosurgical Intensive Care Unit (RNICU) during 1994-
2000. $2^{\circ}$ LOC was defined as a decrease in Glasgow Coma Score (GCS) $\geqslant 2$ points occurring at any time following the injury.

Results: 121 children were admitted to the RNICU over the study period. The median age was 8 years (range $0.16-16$ ); 86 were boys, 35 girls. $2^{\circ}$ LOC occurred in $39(32 \%)$. The median GCS prior to the $2^{\circ}$ LOC was 13 (range 7-15) and the median GCS after deterioration 7 (range 3-13). 14/39 (36\%) had an ICH, $10(26 \%)$ had brain contusions, $5(13 \%)$ had a seizure, $4(10 \%)$ had diffuse cerebral swelling, $2(5 \%)$ had a respiratory arrest and a definite cause for the 2 LOC was not established in $4(10 \%)$. 35/39 $(90 \%)$ were symptomatic at 6 hours but $4 / 39(10 \%)$ were not. All 4 children with a $2^{\circ} \mathrm{LOC}$ on who were asymptomatic at 6 hours had $\mathrm{ICH}$; only $2 / 4$ had a skull fracture. The GCS fell to $\leqslant 4$ in all 4 cases; 3 had a respiratory arrest and required intubation.

Conclusion: These data suggest that discharging TBI children who are asymptomatic at 6 hours may allow home patients who subsequently develop a serious complication. If children are discharged early it is essential that parents are aware of the need to return to hospital immediately if symptoms develop.

\section{P1 1 SELECTIVE HEAD COOLING PROTECTS THE BRAIN IN A PIGLET MODEL OF HYPOXIC ISCHAEMIC BRAIN INJURY}

J. Tooley', S. Satas', H. Porter', I.A. Silver ${ }^{3}$, M. Thoresen'. 'Dept of Child Health, St Michaels Hospital, University of Bristol; ' ${ }^{2}$ Dept of Pathology, St Michaels Hospital, University of Bristol; ${ }^{3}$ Dept of Anatomy, School of Veterinary Science, University of Bristol, UK

Background: Selective head cooling $(\mathrm{SHC})$ may be therapeutic in the management of neonatal hypoxic ischaemic (HI) brain injury. It is proposed that $\mathrm{SHC}$ combined with mild total body hypothermia enhances local neuroprotection whilst minimising the occurrence of systemic side effects.

Aim: To determine whether SHC combined with mild total body cooling for a period of 24 hours reduces cerebral damage in our piglet survival model of global $\mathrm{HI}$.

Method: Eighteen anaesthetised piglets (median age $18 \mathrm{hrs}$ ) received a $45 \mathrm{~min}$ global $\mathrm{HI}$ insult (the severity of which is judged by the length of time the EEG amplitude is below <6iv) followed by randomisation to either remain normothermic (NT) or to receive SHC using a cap perfused with cold water to maintain rectal temperature $\left(T_{\text {rectal }}\right)$ at $34-35^{\circ} \mathrm{C}$ for $24 \mathrm{hrs}$ following the $\mathrm{HI}$ insult. Pigs in the NT group had a median $T_{\text {ral }}$ of $39.1^{\circ} \mathrm{C}$ (Interquartile range 38.9$39.1^{\circ} \mathrm{C}$ ) for the $24 \mathrm{hrs}$ following the insult, whilst piglets in the SHC group were cooled to a $\mathrm{T}_{\text {rectol }}$ of $34.8^{\circ} \mathrm{C}\left(\mathrm{IQR} 34.5-35.0^{\circ} \mathrm{C}\right)$ during this period. Anaesthesia was stopped $24 \mathrm{hrs}$ after the insult. Neurological assessments scores ( 0 abnormal-22 normal) were performed before at 30,48 , and 72 hrs after the insult. At 72 hrs the pigs were anaesthetised and the brains perfusion fixed for neuropathological assessment using light microscopy. Six main regions of the brain were scored $0(0 \%)-4$ (>75\% damage) (Total brain neuropathology score 0-24).

Results: The severity of the $\mathrm{HI}$ insult was similar in the SHC vs NT group $(25.2 / 45 \mathrm{~min}$ vs $29.7 / 45 \mathrm{~min}(\mathrm{p}=0.29)$ of low $E E G$ amplitude). The mean neurology scores at 30 and 48 hours were significantly better in the SHC group vs the NT group $(7.1$ vs 2.7 $(p=0.02))(14.2$ vs $6.4(p=0.02))$ respectively. The mean total neuropathology score was lower in the SHC group compared to the NT group (3.5 vs 9.9 ( $p=0.037)$ ). Regional pathology scores demonstrated significantly less damage in the SHC group in both the cortex (1.1 vs $2.3(p=0.041))$ and basal ganglia $(0.4$ vs $1.9(p=0.019))$.

Conclusions: Selective head cooling combined with mild systemic hypothermia is neuroprotective when started immediately after the insult in our piglet model of HIE.

\section{P12 HOW ACCURATE IS THE CEREBRAL FUNCTION MONITOR FOR NEONATAL SEIZURE DETECTION?}

J.M. Rennie', G. Wilson', G.B. Boylan', R. Pressler², R. Hooper'². Departments of 'Child Health, ${ }^{2}$ Neurophysiology and ${ }^{3}$ Public Health Sciences, King's College Hospital, London

Introduction: The cerebral function monitor (CFM) is widely used in neonatal intensive care units to detect seizures. It is not known how well this method compares with standard EEG.

Aim: To determine the accuracy of CFM monitoring by comparing it to simultaneous EEG and to assess inter-observer agreement of CFM seizure detection. 
Methods: Video-EEG was used to detect seizures in babies admitted to the NICU. Each baby had CFM recordings at speeds of 6, 15, and $30 \mathrm{cms} / \mathrm{hr}$ during the EEG. Four neonatologists, trained in CFM seizure recognition independently rated one-hour CFM samples at three speeds from each baby. Inter-observer agreement was quantified using Cohen's kappa.

Results: CFM traces from 20 babies with EEG seizures and 20 babies without EEG seizures were analysed. Overall the CFM performed poorly as a seizure detector when compared to simultaneous EEG (sensitivities $38 \%$ at $6 \mathrm{~cm} / \mathrm{hr} ; 54 \%$ at $15 \mathrm{~cm} / \mathrm{hr} ; 55 \%$ at $30 \mathrm{~cm} / \mathrm{hr}$ ). Agreement between observers was not good at any speed but was best at a speed of $30 \mathrm{~cm} / \mathrm{hr}$ (kappa 0.38 ). The CFM usually detected seizures that were generalised but frequently missed seizures that were focal, low amplitude or shorter than one minute in duration.

Conclusion: Our results show that approximately half of all neonatal seizures may be missed using CFM alone. Neonatal seizures need to be diagnosed, characterised and quantified first using EEG. The CFM may then be useful for long term monitoring.

\section{P13 RISK FACTORS FOR EARLY ONSET GROUP B STREPTOCOCCAL SEPSIS; A CASE CONTROL STUDY}

S.J. Oddie, N.D. Embleton. Neonatal Unit, Royal Victoria Infirmary, Newcastle on Tyne

Objectives: To quantify risk factors for, and the prevalence of, early onset group B streptococcal sepsis in the former Northern health region of the UK.

Design: Cases of early onset (first week) group B streptococcal sepsis were collected prospectively for two years from April 1998 and compared to four controls each, matched for time and place of delivery.

Results: The prevalence of early onset group B streptococcal sepsis was 0.57 per 1000 livebirths. Premature infants were $38 \%$ of all cases and $83 \%$ of the deaths. Prematurity lodds ratio $10.4,95 \%$ confidence interval 3.9-27.6), rupture of the membranes $>18$ hours (OR $25.8,95 \% \mathrm{Cl} 10.2-64.8)$, rupture of the membranes before the onset of labour (OR $11.1,95 \% \mathrm{Cl} 4.8-25.9$ ) and intrapartum fever (OR $10.0,95 \% \mathrm{Cl} 2.4-40.8)$ were significant risk factors for infection. Had the PHLS interim best practice recommendations been uniformly applied, 29 of 37 cases $(78 \%)$ might have had their disease prevented or ameliorated. At least 23 of these $29(79 \%)$ could have had antibiotics for 4 hours or more before delivery. In achieving this, approximately $16 \%$ of all women would have been given intrapartum antibiotics. Had prophylaxis been offered on the basis of the presence of prematurity or rupture of the membranes before the onset of labour, $25(71 \%)$ of cases could have been treated (OR $14.495 \% \mathrm{Cl}$ $5.6-38.1) .21$ of these $25(84 \%)$ would have been in hospital for long enough to have had four hours of antibiotics before delivery.

Conclusions: Risk factor based prevention of early onset group B streptococcal infection might reduce the prevalence of infection at the cost of treating a significant number of women with risk factors. The use of rupture of the membranes before the onset of labour as a risk factor might be expected to improve the success of prophylaxis guidelines.

\section{P14 DOES THE DISTANCE FROM PAEDIATRIC ONCOLOGY CENTRES AFFECT THE QUALITY OF LIFE OF FAMILIES WITH CHILDREN ON CANCER CHEMOTHERAPY?}

P.J. Kearney, M. O'Reilly, A. McNinch, T. Parkyn, M.G. Mott. Departments of Paediatrics and Child Health and Applied Social Studies, University College, Cork; Paediatric Unit, Royal Devon and Exeter Hospital; Department of Paediatric Oncology, University of Bristol

Aims: The aims of this project were to explore, describe and understand the experience of travelling to paediatric oncology centres, and to identify the burdens of distance for the families of children with cancer and leukaemia.

Methods: This initial exploration of family experiences required a qualitative methodology. Two paediatric units associated with centres that had different policies of decentralising care cooperated with the study. Parents were purposefully selected and invited to participate. Four focus group interviews moderated by an experienced sociologist were recorded. A total of 22 parents ( 5 fathers and 17 mothers) were interviewed. The transcripts were analysed using a grounded theory approach.

Results: The experience of having a child with cancer or leukaemia was very intense for both groups, but there were differences related to the burden of travel. Travel was hardly an issue for the decentralised group. The parents using a more centralised service identified several burdens of travel. They were exhausted by the uncertainty of travel arrangements and the huge distances involved because of repetitive round trips. The experience of travelling with a sick child was frightening and aggravated by the worry of car accidents and the risk of infection in crowded trains. Some of the children's behaviour was barely tolerable during travel. Serious financial problems resulted from the need for a second car, accommodation near the centre, and time lost from work. Family disruption was more severe for the families associated with the centralised service and most parents were very aware of sibling neglect.

Conclusions: The recommendation of one paediatric oncology centre per 5 million population remains suitable in dense population areas, where parents are within commuting distance. Maximal devolution of care reduces the burden of travel on families and provides a better solution than centralisation in less densely populated areas. There is a need for appropriate standards of staffing and accommodation for units with maximal devolution of care.

\section{P15 INCLUDING HIV IN THE INTEGRATED MANAGEMENT OF CHILDHOOD ILLNESS: AN EVALUATION OF AN ALGORITHM FOR USE BY PRIMARY HEALTH CARE WORKERS TO DETECT SYMPTOMATIC HIV IN CHILDREN}

S. Liebeschuetz, C. Horwood, D. Blauuw, S. Cassol. Ngwelezana Hospital, kwaZulu-Natal, South Africa

Aims: The Integrated Management of Childhood Illness (IMCI) is a WHO/UNICEF strategy aimed at reducing mortality and morbidity in children by improving care at primary level. AIDS is a leading cause of death in children in southern Africa and primary level healthworkers urgently need skills to detect and care for these children. To achieve this, diagnosis and management of symptomatic HIV infection was added to the existing $\mathrm{IMCl}$ algorithm. We evaluated the validity of this component of the algorithm in detecting children with symptomatic HIV infection.

Methods: Children attending the outpatients department in a district hospital in South Africa were assessed using the HIV algorithm and by a paediatrician. All children were then tested anonymously for HIV. The validity of the algorithm in detecting symptomatic HIV was compared to a paediatrician and clinical data was used to develop an improved algorithm.

Results: Of 690 children enrolled in the study 198 (28.7\%) were HIV infected. The algorithm correctly identified $56 \%$ of HIV infected children compared to $72 \%$ by the paediatrician. Odds ratios were calculated to identify the most sensitive clinical predictors of HIV infection and an improved algorithm was developed, with a sensitivity of $65 \%$ and a specificity of $85 \%$.

Conclusions: HIV disease can be effectively diagnosed by primary level health workers using an algorithmic approach. An improved HIV algorithm developed from this study has been adopted by WHO for use in countries with high HIV prevalence and will be used by $I \mathrm{MCl}$ practitioners thoughout southern Africa to identify and care for HIV infected children.

\section{P16 THE ROLE OF A NON-CULTURE TECHNIQUE (BROAD-RANGE 16S PCR) IN PATHOGEN IDENTIFICATION IN EMPYEMA THORACIS}

S. Saglani', K. Harris², J. Hartley², C. Wallis'. Dept of 'Respiratory Medicine and ${ }^{2}$ Microbiology, Great Ormond Street Hospital, Great Ormond Street, London

The incidence of childhood empyema thoracis is increasing and may be attributable to unusual organisms. However, pleural fluid is frequently sterile on culture, because of previous antibiotic therapy. PCR detection of bacterial genes has improved diagnosis in many microbial infections. A broad-range PCR that detects the bacterial $16 \mathrm{~S}$ gene has been developed for the identification of causal organisms in empyema thoracis.

Aims: 1 To assess the increase in diagnostic yield using 16S PCR of pleural fluid from children with empyema. 2 To assess the range of organisms isolated, and to compare concordance of organism isolation using traditional culture and molecular techniques.

Method: All children admitted between January 2000 and October 2001, allocated an ICD-10 diagnosis of empyema were reviewed. Pleural fluid was investigated by microbial culture and $16 \mathrm{~S}$ PCR 


\begin{tabular}{|llll|}
\hline Abstract P19 & & \\
\hline & $\begin{array}{l}\text { Live \& family work } \\
\text { on farm \%(n) }\end{array}$ & $\begin{array}{l}\text { Work on farm } \\
\text { only \%(n) }\end{array}$ & Controls \%(n) \\
\hline Ever wheeze/whistle** & $24.8(131)$ & $30.2(85)$ & $32.5(1243)$ \\
Doctor diagnosed asthma* & $17.4(92)$ & $21.3(60)$ & $22.2(847)$ \\
Ever itchy rash*** & $30.1(159)$ & $35.2(99)$ & $38.4(1466)$ \\
Doctor diagnosed eczema*** & $23.1(119)$ & $28.8(80)$ & $31.1(1164)$ \\
Ever sneezing with out flu*** & $12.8(68)$ & $17.8(50)$ & $21.6(828)$ \\
Doctor diagnosed hay fever*** & $8.1(43)$ & $10.3(29)$ & $14.2(542)$ \\
Skin prick positive* & $19.4(49)$ & $27.3(33)$ & $29.4(126)$ \\
\hline${ }^{*} \mathbf{P}<0.05, * * P<0.01, * * * P<0.001$ & & & \\
\hline
\end{tabular}

Results: Forty-one patients were included in the analysis. Mean age was 5.27 years. Twelve $(27.9 \%)$ patients had pleural aspiration at the referring hospital, $4(33.3 \%)$ of which were culture positive. A median of 8.4 days of antibiotic therapy was given prior to pleural aspiration at our centre. Pleural samples were sent for culture from all 41 patients, $6(14.6 \%)$, were positive. Twenty-seven patients also had $16 S$ PCR analysis done. Of these, 17 (63.0\%), had an organism detected on PCR, compared to only $4(14.8 \%)$ being culture positive $(p=0.019)$. Eleven $(40.7 \%)$ patients had an organism identified only by $P C R$, and from no other samples. The additional organisms detected were Strep.pneumoniae (8), strep pyogenes (2), Fusobacterium sp (1). There was 100\% concordance in organisms identified using PCR \& culture from samples analysed either at the local hospital or at our centre.

Conclusion: Significantly more organisms were identified using this molecular technique than with traditional culture. Broad-range 16 sPCR is a valuable and reliable additional tool for identification of causal organisms in empyema thoracis. A result can be obtained in forty-eight hours.

\section{P17 PARENTAL ATTITUDES TO ASTHMA TREATMENT: THEIR SIGNIFICANCE FOR MEDICATION ADMINISTRATION AND OUTCOME OF AN EDUCATIONAL INTERVENTION}

J.Y. Paton, A-M. Love, C. Hart, N. Gibson, M. Morgan'. Royal Hospital for Sick Children, Glasgow; 'King's College, London

Aims: We investigated reasons for non-compliance with inhaled preventive treatment and the prevalence of intentional and unintentional non-compliance among parents of pre-school children with asthma attending a specialist respiratory clinic. In a randomised control trial, we then evaluated the effectiveness of an intervention (parent-centred booklet and consultation) designed to respond to parental beliefs and anxieties about asthma and its treatment likely to lead to non-compliance.

Design: 83 children were studied for a baseline period of 3 months using objective monitoring of inhaler via an electronic inhaler timer and parent symptom diary. 44 were then randomised to the intervention and 39 to receive usual care. Both groups were then followed for a further 3-months using objective monitoring.

Results: Measured compliance was low with only $29 \%$ before and $24 \%$ after the intervention taking more than $80 \%$ of the recommended doses; $44 \%$ before the intervention, and $42 \%$ after, took less than $40 \%$ of the recommended doses. At the first interview, reasons identified for non-compliance included problems giving medication $(43 \%)$, forgetting $(66 \%)$ and changing the dose or timing $(30 \%)$. On follow-up there was no difference in measured compliance in the group receiving the intervention, despite a high level of satisfaction with both the intervention booklet and consultation.

Conclusion: This study has found a high level of non-compliance with inhaled preventer medication in parents of pre-school children. Compliance did not improve after an educational intervention targeted at parent's concerns and anxieties both about asthma and the medications used to treat it. Among this relatively disadvantaged population, there was evidence that the circumstances and demands of everyday life had a more major influence on compliance.

\section{P18 SALBUTAMOL BOLUS VS AMINOPHYLLINE INFUSION IN SEVERE ASTHMA: A RCT}

G. Roberts ${ }^{1,2}$, D Newsom ${ }^{3}$, K Gomez ${ }^{2}$, A Thomas ${ }^{2}$, A Raffles ${ }^{4}$, S Saglani ${ }^{4}$,

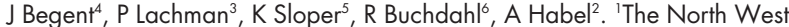
Thames Asthma Study Group (Imperial College School of Medicine at St
Mary's, and ${ }^{2}$ West Middlesex, ${ }^{3}$ Northwick Park, ${ }^{4}$ Queen Elizabeth II, ${ }^{5}$ Ealing and ${ }^{6} \mathrm{Hill}$ ingdon Hospitals)

Introduction: In severe acute asthma, it is unclear which is the most effective intravenous bronchodilator, aminophylline or salbutamol. It has also been reported that salbutamol need only be given as a single bolus [Browne, Lancet 1997]. We have compared a salbutamol bolus with an aminophylline infusion in severe acute asthma in a randomized, double-blinded design.

Methods: Children (1-16y) with acute severe asthma (Asthma Severity Score (ASS) $\geqslant 7$ on 9-point scale [Bishop, J Clin Epi 1992]), presenting to 5 district hospitals, received systemic steroids and 3 back-to-back nebulizers of salbutamol and ipratropium. Those with little improvement (change in ASS $\leqslant 2$, total ASS $\geqslant 7$ or requiring oxygen) were randomized to receive either a short salbutamol bolus $(15 \mathrm{mcg} / \mathrm{kg}$ over 20 minutes) followed by a saline infusion or a continuous aminophylline infusion $15 \mathrm{mg} / \mathrm{kg}$ over 20 minutes then $0.9 \mathrm{mg} / \mathrm{kg} / \mathrm{h}$ ). Both groups continued to receive nebulized therapy. Those with life-threatening features were excluded. The subject's course (ASS, oxygen requirement) was followed until discharge. Ethical committee approval and informed consent were obtained.

Results: Eighteen $(41 \%)$ children were randomized to salbutamol and twenty-six $(59 \%)$ to aminophylline. The two groups were well matched at baseline (eg age and severity). An intention to treat analysis revealed a similar improvement in ASS to 2 hours (difference $-0.08,95 \% \mathrm{Cl}-0.97$ to 0.80 ); a trend towards a longer duration of oxygen therapy in the salbutamol group $(2.56$ times longer, $95 \% \mathrm{Cl}$ 0.92 to $7.18, \mathrm{P}=0.07)$; and a significantly longer time to discharge in the salbutamol group $(1.49$ times longer, $95 \% \mathrm{Cl} 1.06$ to 2.10 , $\mathrm{P}=0.02)$. Five $(28 \%)$ salbutamol and six $(23 \%)$ aminophylline subjects required additional intravenous therapy.

Conclusions: An aminophylline infusion and a single salbutamol bolus were equally effective after two hours of therapy. An aminophylline infusion was the superior therapy in terms of length of admission. Funding provided by Thames NHS Responsiveness Funding Programme.

\section{P19 INITIAL FINDINGS OF THE STUDY OF ASTHMA AND ALLERGY IN SHROPSHIRE}

M.R. Perkin, D.P. Strachan. Department of Public Health Sciences, St George's Hospital Medical School, London

Aims: To determine whether farmer's children in the UK have a reduced prevalence of allergy and to investigate potential protective mechanisms.

Methods: Initial questionnaire survey of allergy prevalence to 7226 primary school children in rural Shropshire: 4785 replied $(66 \%)$. All the farmer's children and a random sample of the other children (1458 children in total) were invited for a number of allergy tests. 937 consented $(64 \%)$ of whom $879(60 \%)$ participated. The tests included skin prick testing with the following allergens: dog, cat, horse and cow hair, grass mix, house dust and 3 storage mites (Acarus siro, Lepidoglyphus destructor \& Tyrophagus putrescentiae).

Results: In the table, the farmer's children are divided into two groups depending on whether the child lives on a farm or not.

Conclusions: This is the first study to confirm that farmer's children in the UK have a reduced risk of atopy. The protective effect for eczema has not previously been observed. The protection is apparent on both subjective (questionnaire) and objective (skin prick) tests. No obvious difference in protective effect was seen between arable, mixed and livestock farmer's children. The study will allow a number of dietary and environmental factors (including domestic endotoxin and allergen levels) to be reviewed. 


\section{P20 THE ROLE OF HEALTHCARE DELIVERY IN THE OUTCOME OF MENINGOCOCCAL SEPTICAEMIA IN CHILDREN}

N. Ninis, C. Phillips, L. Bailey, S. Nadel, J. Britto, I. Maconochie, J.I. Pollock, A. Winrow, P.G. Coen, R. Booy, M. Levin. Department of Paediatrics ICSTM / Research Unit RCPCH

Aims: Meningococcal Disease (MD) is the commonest infectious causes of death in children in the United Kingdom and provides a clearly defined patient group in which to test the hypothesis that outcome is dependent on the quality of health care delivery.

Methods: In order to establish if sub-optimal management contributes to poor outcome we undertook a national case-control study of childhood deaths from meningococcal disease. For each death three survivors from the same region of the country were sought. Predefined and widely accepted criteria were used to define optimal diagnosis and management of meningococcal disease. A panel of paediatricians blinded to the outcome assessed case records using a standardised proforma and scored patients for departures from this optimal management.

Results: 143 cases and 355 controls were included. Departures from ideal (protocolized) management occurred more frequently in the cases than in the controls The risk of death increased with the number of departures from ideal care, the odds ratio for death being $4.51 \mathrm{lCl}$ 2.5-8.3) with one failing increasing to $33(\mathrm{Cl} 6.6158)$ with 5 failings. Multivariate analysis identified 3 factors that were independently associated with death: i) Failure to recognise complications ii) inappropriate fluid therapy iii) other failure including inadequate inotrope, ventilation errors and interhospital transport problems.

Conclusions Suboptimal health care delivery significantly reduces the likelihood of survival in children with meningococcal disease. Improved training of medical and nursing staff, adherence to published protocols and increased consultant supervision may improve the outcome for children not only with meningococcal disease but other life threatening illnesses.

\section{P21 HERBAL CREAMS USED FOR ATOPIC ECZEMA IN BIRMINGHAM,UK CONTAIN POTENT CORTICOSTEROIDS}

H.M. Ramsay, W. Goddard, S. Gill, C. Moss. Department of Dermatology, Birmingham Children's Hospital, Steelhouse Lane, Birmingham, B4 6NH and West Midlands Quality Control Laboratory, City Hospital, Birmingham B18 $7 Q H$

Herbal creams for eczema are readily available to the public from unregulated sources. A previous report of steroid contamination of herbal creams' raised our suspicions about "miracle creams" for eczema reported by families attending our paediatric skin clinic. Treatment may only be reported when dependance has developed, continued supply causing financial embarrassment. Children may be unwittingly using potent topical steroids, particularly since such products are perceived as safe and suitable for the face.

Aims: To analyse the corticosteroid content of 'herbal' creams reported as effective for the treatment of atopic eczema by patients attending the paediatric dermatology clinic.

Methods: Patients attending the paediatric dermatology clinic between June and September 2001 who reported using herbal creams with good effect for atopic eczema were asked to submit the cream for analysis using high pressure liquid chromatography. Hydrocortisone, clobetasone, betamethasone valerate and clobetasol proprionate were used as standards.

Results: 12 different creams from 6 patients, median age 4 years (range 6 months-13 years) were analysed. 7 (58\%) contained corticosteroid; hydrocortisone (2), betamethasone valerate (1), clobetasol proprionate (2), clobetasol proprionate + hydrocortisone (1), clobetasol prorionate + betamethasone valerate (1).

Conclusions: The majority of herbal creams submitted for analysis contained corticosteroids; over half contained the most potent preparation, clobetasol proprionate (Dermovate $\left.{ }^{\circledR}\right)$. Potent or very potent topical steroids can cause irreversible skin atrophy and suppression of the hypothalamic-pituitary-adrenal axis particularly in children. Parents were unaware that they contained steroid and regarded them as safe for use on their child's face. There is an urgent need for tighter regulation of suppliers of 'herbal' creams and for increased public education about the potential dangers of alternative therapies.

1. Keane FM, et al. Analysis of Chinese herbal creams prescribed for dermatological conditions. BM 1999;318:563-4.

\section{P22 DOES WAFTING WORK? THE EFFICACY OF NON CONTACT OXYGEN DELIVERY SYSTEMS}

P. Davies, D. Cheng, A. Fox, L. Lee. Luton and Dunstable NHS Trust, UK

Aims: Wafting oxygen is a strategy to deliver oxygen $\left(\mathrm{O}_{2}\right)$ to a patient who may not tolerate delivery systems which involve contact on the face. Whilst improvement in percutaneous $\mathrm{O}_{2}$ saturations have been observed, the percentage of $\mathrm{O}_{2}$ delivered has never been quantified. We wished to assess (1) the concentration of $\mathrm{O}_{2}$ delivered to a patient and (2) the area in which this applies, with various methods of wafting $\mathrm{O}_{2}$.

Methods: Three methods of wafting $\mathrm{O}_{2}$ were examined; an Ambu bag and mask, a standard paediatric Hudson $\mathrm{RCl}$ face mask, and a piece of standard green oxygen tubing. In each case the delivery system was fixed, and contour lines for $\mathrm{O}_{2}$ concentrations of $30 \%$ to $70 \%$ in $10 \%$ intervals were found with a Teledyne oxygen meter, at an $\mathrm{O}_{2}$ flow rate of $5 \mathrm{I} / \mathrm{min}$ and $10 \mathrm{l} / \mathrm{min}$. Experimental conditions simulated an infant in a cot in a paediatric ward.

Results: The Ambu bag and mask are not recommended for wafting $\mathrm{O}_{2}$ delivery, as the flow back valve may close and result in insignificant gas flow. Oxygen tubing delivered $\mathrm{O}_{2}$ to an area too narrow for use with an active patient. The Hudson RCl face mask, delivered $\mathrm{O}_{2}$ to a large area. The contour lines for the $\mathrm{O}_{2}$ tubing and face mask systems are presented in figure 1.

Conclusions: In certain clinical situations wafting oxygen through a standard paediatric face mask can deliver $30-40 \% \mathrm{O}_{2}$ in a large area without irritating the patient. Care should be taken to place the mask in the area described (ie opposite the sternum) to give the maximum benefit. In the short term (for feeding or neonatal resuscitation), $\mathrm{O}_{2}$ can be delivered with standard oxygen tubing aimed at the airway.

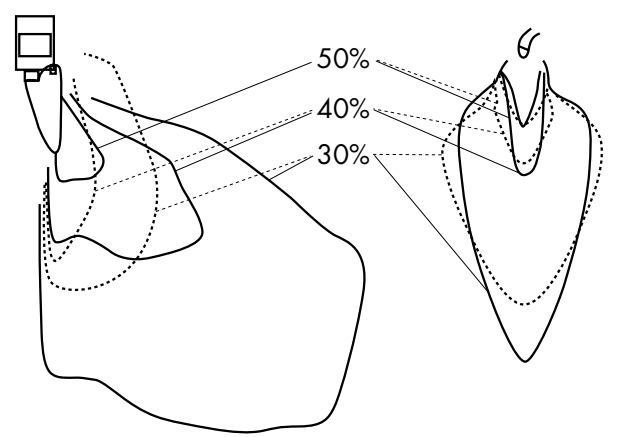

Abstract P22 Contour lines of $\mathrm{O}_{2}$ delivery with the Hudson Face mask (on left) and oxygen tubing internal diameter $5 \mathrm{~mm}$ (on right). Solid line $=10 \mathrm{l} / \mathrm{min}$ flow; dotted $=5 \mathrm{l} / \mathrm{min}$. Scale: each box $=5 \times 5$ $\mathrm{cm}$.

\section{P23 PODOCYTES DEMONSTRATE FEATURES OF INSULIN RESPONSIVE GLUCOSE UTILIZATION AND ACTIN RE-ORGANISATION-A POTENTIAL EXPLANATION FOR DIABETIC MICROALBUMINURIA}

Coward RIM', Welsh $\mathrm{Gl}^{2}, \mathrm{Ni}^{1} \mathrm{~L}^{1}$, Bai X', Tavaré $\mathrm{JM}^{2}$, Mathieson PW', Saleem MA'. 'Academic Renal Unit, Southmead hospital, Bristol and 'Department of Biochemistry, University of Bristol.

Diabetic nephropathy is the leading cause of end-stage renal failure globally, and is also increasing in the childhood population. The mechanisms by which microalbuminuria and subsequent glomerulosclerosis occur are unknown. The specific physiological means that cells of a high energy requirement utilize glucose is by the insulin sensitive glucose transporter, GLUT4. The expression of GLUT4 is limited, primarily to skeletal muscle and adipose cells, and it has been thought that the glomerulus does not display insulin responsiveness. Using a cell line of human conditionally immortalised podocytes, we have demonstrated expression of GLUT4 and its membrane partner VAMP2, with immunofluorescence (IF) and Western blotting. We have examined functional changes of the podocyte in response to glucose $(25 \mathrm{mmol})$ and/or insulin $(250 \mathrm{nmol})$ at 15 minutes by IF, radiolabelled glucose uptake assays and real time micro-injected GFP-GLUT4 tagged imaging of the cells. These demonstrate that GLUT4 vesicles move from the cytoplasm to the cell membrane in response to glucose, but insulin is required for docking and increased 
cellular glucose uptake (factor 2.1, $\mathrm{p}<0.01$ ). The cytoskeleton is crucial in this process with F-actin reorganising to a cortical distribution with stimulation of the cells and abrogation of the pathway when actin is disrupted by Cytocholasin D. Thus we have shown that the podocyte is a novel glucose utilizing, insulin sensitive cell, with actin redistribution in response to insulin and glucose. Because of the known role of F-actin in supporting podocyte foot processes, we suggest that metabolic disturbances of insulin and glucose in diabetes lead to structural foot process alteration, and hence microalbuminuria.

\section{P24 EARLY NASAL CONTINUOUS POSITIVE AIRWAYS PRESSURE (NCPAP) WITH PROPHYLACTIC SURFACTANT FOR NEONATES AT RISK OF RDS. THE} IFDAS MULTI-CENTRE RANDOMISED TRIAL.

Thomson MA, on behalf of the IFDAS Study Group. Department of Paediatrics and Neonatal Medicine, Hammersmith Hospital, London, UK.

Aims: To investigate whether early nCPAP (Infant Flow Driver ${ }^{\mathrm{TM}}$ ) following prophylactic surfactant (Curosurf®) would reduce the need for mechanical ventilation in neonates at risk of RDS and subsequent CLD.

Methods: In a prospective multicentre trial, inborn infants 27-29 wks were randomised before birth to one of 4 treatment arms, early nCPAP with prophylactic surfactant (gpl), early nCPAP +/- rescue surfactant (gp2), early IPPV with prophylactic surfactant (gp3), and conventional management, (IPPV +/- rescue surfactant) (gp4). Results were analysed on an intention to treat basis. Ethical permission was obtained.

Results: 237 babies were enrolled, gp 1 50, gp2 63, gp3 55, gp4 69. No differences in maternal factors, birthweight and gestational age were seen, antenatal steroid use was high (97\%). Surfactant was given to all in gps $1+3,48 \%$ in gp2 and $61 \%$ in gp4. $88(78 \%)$ of infants in gpl +2 were established on nCPAP by 6 hours of age $(p<0.001)$. Time to wean (median $+I Q$ range) was shorter in gp2 20 min (0-95), versus $80 \mathrm{~min}(10-155)$ for gpl $(p=0.01)$. Increasing gestational age increased the probability of success $(p<0.001)$. The requirement for mechanical ventilation in the first 5 days (120hr) of life was highest in gp3 47 (85\%), followed by gp4 40 (58\%), gp2 22 $(35 \%)$ and lowest in gpl $17(34 \%)$. The total duration of ventilation in the first 5 days (median + IQ range) was gp 1120 min (30-540), gp2 $67.5 \mathrm{~min}(2.5-2310)$, gp3 $1440 \mathrm{~min}$ (480-5160) and gp4 $720 \mathrm{~min}$ $(0-4320) \quad p=0.001$. Regression analysis showed that increasing gestational age shortened the duration of ventilation. When the total respiratory support (mechanical ventilation + $\mathrm{nCPAP}$ ) until EDD or discharge home (if earlier) was assessed there was not difference between the groups. No difference was found between groups for oxygen dependency at 28 days or 36 weeks gestational age, or in the rates of respiratory, ultrasound and other neonatal complications.

Conclusion: The use of nCPAP with prophylactic surfactant, or nCPAP alone reduced the need for mechanical ventilation when used as initial respiratory support. The effect is influence by gestational age. 